\title{
Four-Dimensional Nonbinary LDPC-Coded Modulation Schemes for Ultra-High-Speed Optical Fiber Communication
}

\author{
Murat Arabaci, Member, IEEE, Ivan B. Djordjevic, Senior Member, IEEE, Lei Xu, and Ting Wang
}

\begin{abstract}
In this letter, we propose a four-dimensional (4-D) nonbinary low-density parity-check-coded modulation (NB-LDPC-CM) scheme suitable for beyond $100-\mathrm{Gb} / \mathrm{s}$ optical fiber communication. Incorporating spectrally efficient modulation formats to achieve high aggregate bit rates and nonbinary LDPC codes for forward error correction (FEC), the proposed scheme offers a superior advanced FEC solution for optical fiber communication systems than the prior-art bit-interleaved LDPC-coded modulation (BI-LDPC-CM) scheme. Compared to the previously reported bit error rate (BER) performance results of BI-LDPC-CM, the proposed scheme offers additional net coding gains (NCGs) of $0.29 \mathrm{~dB}, 1.17 \mathrm{~dB}$, and $2.17 \mathrm{~dB}$ at the BER of $10^{-7}$ when 16-, 32-, and 64-point 4-D constellations are used, respectively.
\end{abstract}

Index Terms-Coded modulation, forward error correction (FEC), low-density parity-check (LDPC) codes, optical fiber communication.

\section{INTRODUCTION}

$\mathbf{T}$ HE IEEE 802.3ba standard on 40/100 Gigabit Ethernet $(40 / 100 \mathrm{GbE})$ was ratified in June 2010 as a response to the ever-increasing demands for higher capacity transmission over optical fiber links. Discussions over the next upgrade for Ethernet have already started. While some argue that 1 Terabit Ethernet ( $\mathrm{TbE})$ should be standardized next to meet the projected demand, some advocate a more conservative upgrade to $400 \mathrm{GbE}$ first. Regardless of whose projections will come true, all agree that $100 \mathrm{GbE}$ is yet another station but not the final destination in the evolution of Ethernet.

As the operating symbol rates increase, the deteriorating effects of fiber nonlinearities and polarization-mode dispersion (PMD) reach levels that inhibit reliable communication over the optical fiber network. Thus solutions for $100 \mathrm{GbE}$ and beyond need to attain ultra high transmission speeds in terms of aggregate bit rates while keeping the operating symbol rates low to facilitate nonlinearity and PMD management. A promising solution employing coded modulation using low-density parity-

Manuscript received April 12, 2011; revised May 29, 2011; accepted June 08, 2011. Date of publication June 20, 2011; date of current version August 19, 2011. This work was supported in part by NEC and in part by NSF under Grants CCF-0952711 and ECCS-0725405.

M. Arabaci and I. B. Djordjevic are with the Department of Electrical and Computer Engineering, University of Arizona, Tucson, AZ 85721 USA (e-mail: arabaci@email.arizona.edu; ivan@email.arizona.edu).

L. Xu and T. Wang are with NEC Laboratories America, Inc., Princeton, NJ 08540 USA (e-mail: leixu@nec-labs.com; ting@nec-labs.com).

Color versions of one or more of the figures in this letter are available online at http://ieeexplore.ieee.org.

Digital Object Identifier 10.1109/LPT.2011.2159968 check (LDPC) codes as component codes has already been discussed in our previous works [1], [2]. The underlying idea is to use spectrally-efficient modulation formats at low symbol rates along with strong LDPC codes for forward error correction (FEC) in order to accomplish reliable communication at high aggregate bit rates.

In addition to spectral efficiency, power efficiency of a modulation format plays an important role in communication system design. Coherent optical communication physically allows using four dimensions for modulation rather than only two dimensions over which conventional modulation formats, e.g., quadrature amplitude modulation, are defined. As a result, one can exploit this four-dimensional (4-D) signal space to set up more power-efficient signal constellations than one could do using the 2-D signal space - by increasing the Euclidean distance between constellation points for a given average signal power [3]. 4-D bit-interleaved LDPC-coded modulation (4-D BI-LDPC-CM) employing binary LDPC codes for FEC was discussed in [2]. In this letter, we employ nonbinary LDPC codes for FEC and show that the proposed 4-D nonbinary LDPC-coded modulation (4-D NB-LDPC-CM) scheme can provide larger coding gains than the corresponding 4-D BI-LDPC-CM scheme. Furthermore, the proposed 4-D NB-LDPC-CM scheme can reduce receiver latency by avoiding costly "turbo-like" iterations between the detection and decoding units, which is essential for acceptable error correction performance in any BI-LDPC-CM scheme.

The remainder of the letter is organized as follows. In Section II, we introduce the proposed 4-D NB-LDPC-CM scheme and highlight its differences from the corresponding prior-art 4-D BI-LDPC-CM scheme. Section III presents our results and discussion. We conclude our letter in Section IV.

\section{FouR-Dimensional NonBINARY LDPC-CODED MODULATION}

Fig. 1 depicts the transmitter and receiver configurations for the proposed 4-D NB-LDPC-CM scheme. Compared to prior-art 4-D BI-LDPC-CM scheme presented in [2], we observe the following differences: 1 ) the set of $m$ binary LDPC encoders are replaced by a single $2^{m}$-ary LDPC encoder, 2) the block interleaver unit, which distributes $N$ codeword bits of each binary LDPC encoder used in BI-LDPC-CM over all of $N$ transmitted symbols [2], is eliminated, 3) the feedback loop into the maximum a posteriori probability (MAP) detector from the decoding unit is eliminated, and 4) symbol-to-bit and vice versa conversion interfaces are eliminated since the nonbinary 


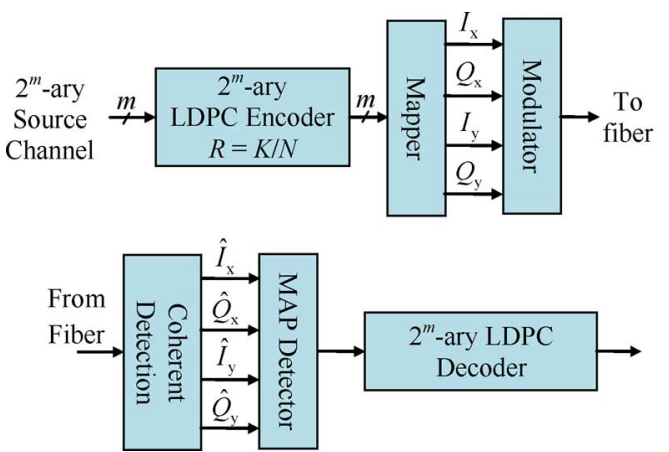

Fig. 1. (a) Transmitter and (b) receiver configurations of a system using 4-D NB-LDPC-CM scheme.

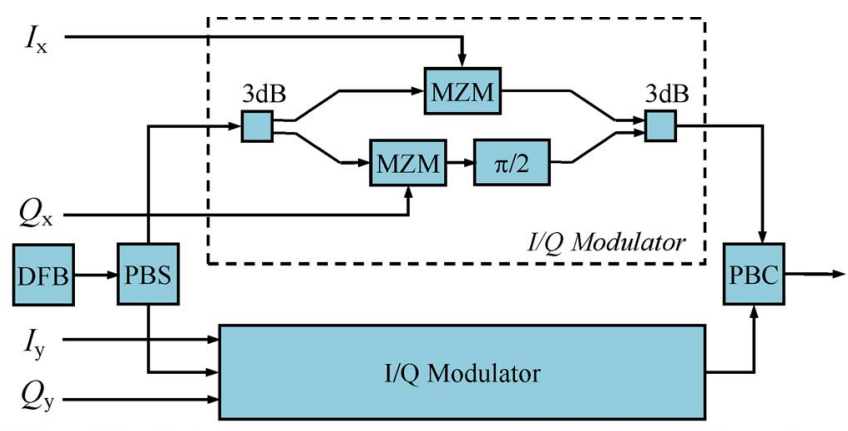

DFB: Distributed feedback laser, PBS/C: Polarization beam splitter/combiner, MZM: Mach-Zehnder modulator, 3dB: 3dB coupler

Fig. 2. Internal structure of a 4-D modulator.

decoder operates at the symbol level. We should highlight item 3 further. BI-LDPC-CM requires iterative detection and decoding for good performance [4]. Since both the MAP detection process and $m$ parallel LDPC decoding processes contribute to computational complexity and latency of the receiver, multiple soft information exchanges between the detection and the decoding units can very fast cause large complexity and latency figures at the receiving ends. Eliminating this feedback loop, the proposed 4-D NB-LDPC-CM scheme can help lower the latency and computational complexity. Moreover, as we will present in Section III, the proposed scheme improves the BER performance.

A $2^{m}$-ary, $m>1, \operatorname{LDPC}(N, K)$ code is an LDPC code of code rate $R=K / N$ defined over the finite field, or the Galois field, of order $2^{m}$, denoted by $\operatorname{GF}\left(2^{m}\right)$. Since every $2^{m}$-ary symbol can be represented as a binary vector of length $m$, we can call $m$ parallel binary source channels as a single $2^{m}$-ary channel as in Fig. 1. In NB-LDPC-CM, an incoming $K$-symbollong input block from a $2^{m}$-ary source channel is encoded into a $2^{m}$-ary LDPC codeword of length $N$. The mapper then maps each $2^{m}$-ary symbol to a point in a signal constellation comprised of $2^{m}$ points. Note that the order of the field over which the component nonbinary LDPC code is designed and the size of the signal constellation are both equal to $2^{m}$. Since they are equal, there is no need for iterative detection and decoding operation in NB-LDPC-CM as mentioned previously. The mapper outputs are then modulated via a 4-D optical modulator whose internal structure is depicted in Fig. 2.
At the receiver, the coherent detector outputs on the two quadratures of the two polarizations are passed to a MAP detector. The MAP detector, which implements the multilevel extension of the well-known Bahl-Cocke-Jelinek-Raviv algorithm as detailed in [5], produces the log-likelihood ratio (LLR) matrix of length $N \times 2^{m}$. Each row $k, 0 \leq k<N$, of the LLR matrix is comprised of $2^{m}$ entries each corresponding to

$$
\lambda\left(\mathbf{s}_{k}^{(a)}\right)=\log \left[\frac{P\left(\mathbf{s}_{k}^{(a)} \mid \mathbf{r}\right)}{P\left(\mathbf{s}_{k}^{(0)} \mid \mathbf{r}\right)}\right],
$$

i.e., the logarithm of the ratio of the conditional probability that the $k$ th symbol sent by the transmitter being the 4-D constellation point $\mathbf{s}_{k}^{(a)}$ corresponding to the $2^{m}$-ary symbol $a \in$ $\mathrm{GF}\left(2^{m}\right)=\left\{0,1, \ldots, 2^{m}-1\right\}$ over the probability that it is being $\mathbf{s}_{k}^{(0)}$ given the received noisy vector $\mathbf{r}$. (As long as the consistency is maintained, any $q$-ary symbol other than the 0 symbol can be used as the reference symbol.) These reliability estimates on each received symbol are then processed by the decoder to determine the codeword sent by the transmitter.

\section{RESUlTS AND DISCUSSION}

In this section, we test the performance of the proposed 4-D NB-LDPC-CM scheme and compare it against the corresponding 4-D BI-LDPC-CM scheme as we vary the constellation sizes and hence the achievable aggregate information bit rates. In our simulations, we assume amplified spontaneous emission (ASE) noise dominated transmission scenario, and assume that chromatic dispersion and PMD are perfectly compensated for. Lastly, we set the symbol rate to 50 Giga-Symbols/s (GS/s).

In 4-D BI-LDPC-CM employing a $2^{m}$-point constellation, we use $m$ identical rate- $0.8 \operatorname{LDPC}(16935,13550)$ codes for FEC. This particular code is chosen due to its good performance under various optical fiber transmission scenarios which were reported previously in [5], [6]. Further details on its construction can also be found in [6]. On the other hand, in the corresponding nonbinary scheme, we use the $2^{m}$-ary rate- 0.8 LDPC $(16935,13548)$ code whose parity-check matrix is constructed by assigning nonzero elements from $\operatorname{GF}\left(2^{m}\right)$ to the $1 \mathrm{~s}$ in the parity-check matrix of the binary LDPC code mentioned above. More information on the construction of nonbinary LDPC codes from binary ones can be found in [7]. In 4-D BI-LDPC-CM, we perform a maximum of 25 decoding iterations and 3 iterative detection and decoding loops. On the other hand, 4-D NB-LDPC-CM performs a maximum of 50 decoding iterations and it does not require any iteration between detection and decoding units. The binary LDPC decoding algorithm is the renowned sum-product algorithm (SPA), and the nonbinary decoding algorithm is the fast Fourier transform (FFT) based nonbinary SPA, whose details can be found in [7] and in the references therein.

Fig. 3 presents our simulation results. (In Fig. 3, optical signal to noise ratio (OSNR) per information bit, i.e., $\mathrm{OSNR}_{b}$, is related to the OSNR per transmitted symbol denoted by $\mathrm{OSNR}_{s}$ via $\mathrm{OSN}_{b}=\mathrm{OSNR}_{s} \cdot\left(\log _{2}(q) \cdot R\right)^{-1}$, which 


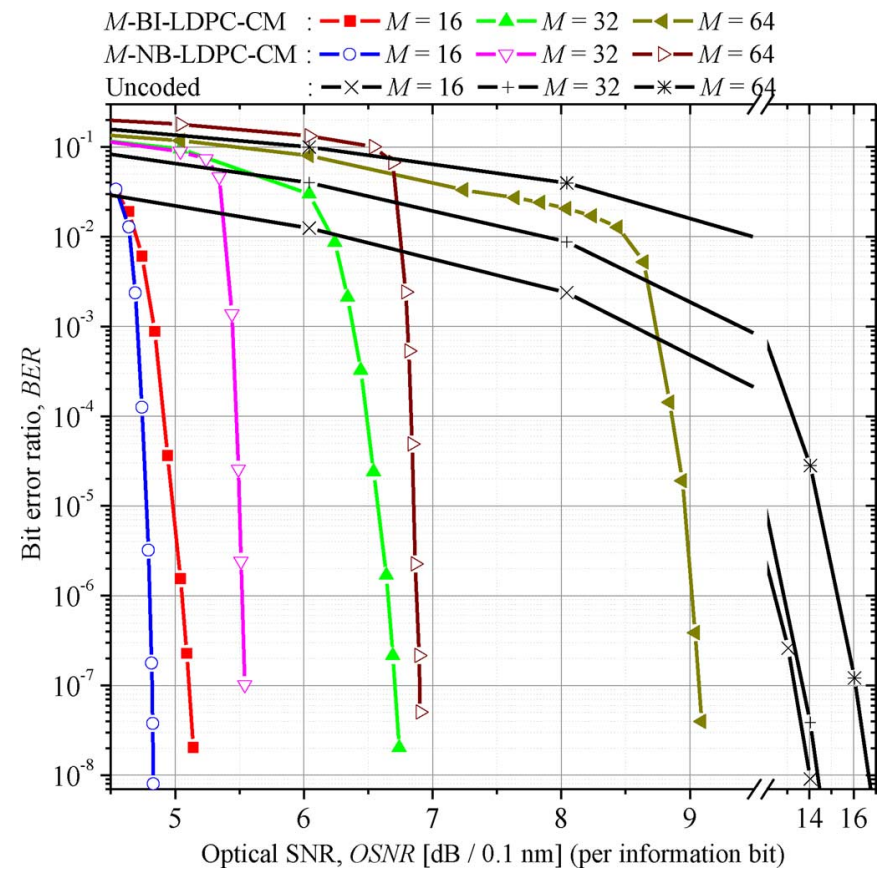

Fig. 3. Performance comparison between 4-D NB-LDPC-CM schemes and the corresponding 4-D BI-LDPC-CM schemes for various constellation sizes.

clearly presents the effects of constellation size and the code rate in $\mathrm{OSNR}_{b}$.) There are three constellation sizes considered, namely 16,32 , and 64 . In order to compare against the 4-D BI-LDPC-CM performance curves presented previously in [2], we use the same signal constellations. Using Fig. 3, we computed the net coding gains (NCGs) for NB-LDPC-CM at the BER of $10^{-7}$ as $8.52 \mathrm{~dB}, 8.21 \mathrm{~dB}$, and $9.14 \mathrm{~dB}$ for 16-, 32-, and 64-point constellations, respectively. As Fig. 3 shows, the proposed scheme outperforms its binary counterpart at all constellation sizes. To elaborate, at the BER of $10^{-7}, 4-\mathrm{D}$ NB-LDPC-CM outperforms 4-D BI-LDPC-CM by $0.29 \mathrm{~dB}$, $1.17 \mathrm{~dB}$, and $2.17 \mathrm{~dB}$ in terms of NCG when 16-, 32-, and 64-point constellations are used, respectively. As the slopes of the performance curves indicate, the gaps between nonbinary and the corresponding binary curves are expected to increase for lower BERs such as $10^{-12}$ and $10^{-15}$, signifying larger NCGs and larger additional coding gains at lower BERs when using the proposed NB-LDPC-CM scheme. We should note that any 4-D constellations other than the ones in [2] can be used. For example, the optimum constellations presented by Sloane et al. [8] may provide an additional $0.5 \mathrm{~dB}$ coding gain at the BER of $10^{-7}$ at the expense of increased implementation complexity since the optimum constellation points require 12digit accuracy, which is much larger compared to accuracy required by the 4-D regular polytopes used in [2]. Since the code rate is fixed at 0.8 , the aggregate information bit rate reaches $160 \mathrm{~Gb} / \mathrm{s}, 200 \mathrm{~Gb} / \mathrm{s}$, and $240 \mathrm{~Gb} / \mathrm{s}$ for 16-, 32-, and 64-point constellations, respectively. At these beyond $100 \mathrm{~Gb} / \mathrm{s}$ transmission rates, NB-LDPC-CM stands out as a stronger advanced FEC scheme.

As a final remark, we observe that additional NCGs provided by the proposed 4-D NB-LDPC-CM scheme over its binary counterpart increases as the underlying constellation size increases. This stems from two reasons. First one is the suboptimality of BI-LDPC-CM [4]. In BI-LDPC-CM, $m$ bits taken from the outputs of $m$ parallel LDPC encoders are mapped to the same transmitted symbol, which forms a dependency between these bits. At the receiver side, however, these dependent bits are decoded independently from one another. To exploit the dependency and to lessen the effects of suboptimality, iterative detection and decoding is employed as alluded to in Section II. As the constellations, and hence the number of bits per symbol, i.e., $m$, grow, the effect of suboptimality gets harder to compensate for. The second reason is related to the "adaptive" nature of NB-LDPC-CM. To elucidate, NB-LDPC-CM matches (or "adapts") the size of the field order to the constellation size; thus, establishes a one-to-one correspondence between $2^{m}$-ary coded symbols and the $2^{m}$-ary constellation points. A $2^{m}$-ary LDPC decoder provides its variable nodes and check nodes with a $2^{m}$-ary state space during decoding. (BI-LDPC-CM provides the binary state space to its variable and check nodes regardless of the number of bits per symbol used during transmission.) In other words, NB-LDPC-CM counteracts the growth in the constellation size by increasing the state space of the nodes used in decoding. As a result, NB-LDPC-CM scheme is able to provide increasing performance margins over the corresponding BI-LDPC-CM scheme as the constellations get larger.

\section{CONCLUSION}

We proposed a nonbinary LDPC-coded modulation scheme, and showed its superior performance over the prior-art BI-LDPC-CM scheme. The superiority of the proposed scheme becomes more pronounced as the underlying constellation sizes and hence the aggregate information bit rates increase. Also, by omitting the iterative detection and decoding loops, which are crucial for good performance in BI-LDPC-CM, the proposed scheme can lower latency and computational complexity at receivers. With these important advantages, we believe the proposed scheme is an outstanding candidate for advanced FEC in future optical fiber communication systems.

\section{REFERENCES}

[1] I. B. Djordjevic et al., "Next generation FEC for high-capacity communication in optical transport networks," J. Lightw. Technol., vol. 27, no. 16, pp. 3518-3530, Aug. 15, 2009.

[2] H. G. Batshon et al., "Ultra high speed optical transmission using subcarrier-multiplexed four-dimensional LDPC-coded modulation," Opt. Express, vol. 18, pp. 20546-20551, Sep. 2010.

[3] H. Bülow and E. Masalkina, "Coded modulation in optical communications," in Proc. OFC, Los Angeles, 2011, Paper OThO1.

[4] J. Hou et al., "Capacity-approaching bandwidth-efficient coded modulation schemes based on low-density parity-check codes," IEEE Trans. Inf. Theory, vol. 49, no. 9, pp. 2141-2155, Sep. 2003.

[5] I. B. Djordjevic et al., "Suppression of fiber nonlinearities and PMD in coded-modulation schemes with coherent detection by using turbo equalization," J. Opt. Commun. Netw., vol. 1, pp. 555-564, Nov. 2009.

[6] I. B. Djordjevic et al., "Large girth low-density parity-check codes for long-haul high-speed optical communications," in Proc. OFC, San Diego, CA, 2008, Paper JWA53.

[7] M. Arabaci et al., "Polarization-multiplexed rate-adaptive nonbinary-quasi-cyclic-LDPC-coded multilevel modulation with coherent detection for optical transport networks," Opt. Express, vol. 18, pp. 1820-1832, Jan. 2010.

[8] N. J. A. Sloane et al., "Minimal-energy clusters of hard spheres," Discrete Computat. Geometry, vol. 14, pp. 237-259, 1995. 